\title{
Subgroup-specific gene expression profiles and mixed epistasis in chronic lymphocytic leukemia
}

\author{
Almut Lütge*1,2,3, Junyan Lu*1, Jennifer Hüllein ${ }^{1}$, Tatjana Walther ${ }^{4}$, Leopold Sellner ${ }^{4,5}$, Bian Wu ${ }^{4,6}$, Richard \\ Rosenquist $^{7,8}$, Christopher C. Oakes ${ }^{9}$, Sascha Dietrich ${ }^{5}$, Wolfgang Huber*1, Thorsten Zenz*4,10
}

1. Genome Biology Unit, EMBL, Heidelberg, 69117, Germany

2. Department of Molecular Life Sciences, University of Zurich, Zurich, Switzerland

3. SIB Swiss Institute of Bioinformatics, University of Zurich, Zurich, Switzerland

4. Molecular Therapy in Hematology and Oncology \& Department of Translational Oncology, NCT and DKFZ, Heidelberg, Germany

5. Department of Medicine V, Heidelberg University Hospital, Heidelberg, Germany

6. Cancer Center, Union Hospital, Tongji Medical College, Huazhong University of Science and Technology, Wuhan 430022, China

7. Department of Molecular Medicine and Surgery, Karolinska Institutet, Stockholm, Sweden

8. Clinical Genetics, Karolinska University Hospital, Solna, Sweden

9. Department of Internal Medicine, Division of Hematology, The Ohio State University, Columbus, USA.

10. Department of Medical Oncology and Hematology, University Hospital Zurich, Zurich, Switzerland.

*contributed equally

\section{Abstract}

Despite the extensive catalogue of recurrent mutations in chronic lymphocytic leukaemia (CLL), the diverse molecular driving events and the resulting range of disease phenotypes remain incompletely understood. To study the molecular heterogeneity of CLL, we performed RNA-sequencing on 184 CLL patient samples. Unsupervised analysis revealed two major independent axes of gene expression variation: the first one aligned with the mutational status of the immunoglobulin heavy variable (IGHV) genes, and concomitantly, with the three-group stratification of CLL by global DNA methylation pattern, and affected biological functions including B- and T-cell receptor signaling. The second one aligned with trisomy 12 status and affected chemokine signaling. Furthermore, we searched for differentially expressed genes associated with gene mutations and copy-number aberrations and detected strong signatures for TP53, BRAF and $S F 3 B 1$, as well as for $\operatorname{del}(11)(q 22.3), \operatorname{del}(17)(p 13)$ and $\operatorname{del}(13)(q 14)$ beyond the dosage effect. We discovered strong non-additive effects (i.e., genetic interactions, or epistasis) of IGHV mutation status and trisomy 12 on multiple phenotypes, including the expression of 893 genes. Multiple types of epistasis were observed, including synergy, buffering, suppression and inversion. Our study reveals previously underappreciated gene expression signatures for (epi)genomic variants in CLL and the presence of epistasis between them. The findings will serve as a reference for a functional resolution of CLL molecular heterogeneity. 


\section{Introduction}

Chronic lymphocytic leukemia (CLL) etiology has been linked to abnormal B-cell receptor (BcR) activation and gene mutations targeting multiple pathways, including DNA damage pathways (TP53, ATM), NOTCH signaling (NOTCH1, FBXW7, MED12) $)^{1-4}$ and the spliceosome $(S F 3 B 1)^{5,6}$. In addition, the IGHV mutation status, the result of a physiological mutation and maturation process, reflects the tumor's cell type of origin and is one of the strongest predictors of clinical behavior ${ }^{7}$. Several genetic subgroups of CLL are known that show profound differences in clinical course, presentation and outcome ${ }^{8,9}$, although considerable variability remains within subgroups.

Gene expression profiling has the potential to provide a better understanding of the functional role of mutations and may help to dissect the disease heterogeneity. Indeed, previous studies of CLL transcriptomes have found substantial variability ${ }^{10,11}$, however, it has been a surprise how little of that variability could be associated with the genetic subgroups or other properties of the disease. For instance, Ferreira et al. ${ }^{10,11}$ found only a few robust gene expression changes associated with the major cancer driver mutations of CLL. IGHV mutation status only accounted for $1.5 \%$ of the overall variance in their study. The study reported two subgroups, termed $\mathrm{C} 1 / \mathrm{C} 2$, as a predictor of clinical outcome independent of known molecular disease groups. A later reanalysis of the data suggested a relation of $\mathrm{C} 1 / \mathrm{C} 2$ to sample processing ${ }^{12}$.

Overall, the relations between prominent genetic events that have significant impact on disease course and the gene expression programmes of CLL have remained unclear. Among possible explanations for this scarcity of associations are small sample sizes, confounding effects of multiple cytogenetic abnormalities or confounding technical variations. More recent studies have thus collected larger cohorts with focus on a particular genetic aberration. Abruzzo et al. ${ }^{13}$ identified a set of dysregulated and potentially targetable pathways in CLL with trisomy 12 . Herling et al. ${ }^{14}$ developed a 17-gene signature that can identify a subset of treatment-naive patients with IGHV-unmutated CLL (U-CLL) who might substantially benefit from treatment with FCR (fludarabine, cyclophosphamide and rituximab) chemoimmunotherapy. These findings underline the importance of transcriptional changes in CLL.

Here, we profiled 184 CLL samples using RNA-sequencing. After careful control of technical variations, and of possible confounding between genetic variants, we searched for transcriptomic signatures and pathway activity changes associated with the major recurrent genetic alternations in CLL. Furthermore, as a step towards gaining a better understanding of functional interdependencies between mutations in a tumor, we 
used a quantitative model of genetic interactions to identify non-additive effects of mutations on gene expression profiles.

\section{Material and Methods}

\section{Data acquisition}

\section{RNA-sequencing}

We selected 184 CLL patient samples for RNA-sequencing. Patients were recruited between 2011-2017 with informed consent. The population was representative for a tertiary referral center. The majority of patients (177 out of 184) showed a typical CLL phenotype and 5 patients were diagnosed with atypical CLL. In total 92 patients had undergone some kind of medical treatment. All patient characteristics are shown in Supplemental Table S1. RNA isolation and library preparation were performed as described before ${ }^{15}$. In short, total RNA was isolated from patient blood samples (CD19+ presorted $n=161)$ using the RNA RNeasy mini kit (Qiagen). RNA quantification was performed with a Qubit 2.0 Fluorometer. RNA integrity was evaluated with an Agilent 2100 Bioanalyzer, and samples with RNA integrity number (RIN) $<8$ were excluded. Sequencing libraries were prepared according to the Illumina TruSeq RNA sample preparation v2 protocol. Samples were paired-end sequenced at the DKFZ Genomics and Proteomics Core Facility. Two to three samples were multiplexed per lane on Illumina HiSeq 2000, Illumina HiSeq3000/4000 or Illumina HiSeqX machines.

Raw RNA-sequencing reads were demultiplexed, and quality control was performed using FastQC ${ }^{16}$ version $^{-10}$ 0.11.5. STAR $^{17}$ version $2.5 .2 \mathrm{a}$ was used to remove adapter sequences and map the reads to the Ensembl human reference genome release 75 (Homo sapiens GRCh37.75). STAR was run in default mode with internal adapter trimming using the clip3pAdapterSeq option. Mapped reads were summarized into per gene counts using htseq-count ${ }^{18}$ version 0.9 .0 with default parameters and union mode. Thus, only reads unambiguously mapping to a single gene were counted. The count data were imported into $\mathrm{R}^{19}$ (version 3.6) for subsequent analysis.

\section{Somatic variants}

Mutation calls for 66 distinct gene mutations and 22 structural variants had been generated in a previous study for 143 out of 184 CLL samples through targeted sequencing, whole-exome sequencing and 
whole-genome sequencing ${ }^{15}$. For the remaining 41 samples, we generated additional targeted and whole-genome sequencing data and called variants using the same pipeline. Statistical analyses were restricted to variants found in at least 5 patients, i.e., to 14 gene mutations (BRAF, NOTCH1, SF3B1, TP53, KRAS, ATM, MED12, EGR2, KLHL6, ACTN2, MGA, NFKBIE, PCLO, XPO1), and 9 copy-number aberrations (CNAs): (trisomy 12, $\operatorname{del}(11)(q 22.3), \operatorname{del}(13)(q 14), \operatorname{del}(17)(p 13), \operatorname{del}(8)(p 12), \operatorname{gain}(8)(q 24)$, gain(2)(p25.3), del(15)(q15.1), gain(14)(q32)) (Supplemental Figure S2B). In addition, the somatic hypermutation status of the immunoglobulin heavy variable (IGHV) and a CLL subtype classification defined by global patterns of $\mathrm{CpG}$ methylation level ${ }^{20,21}$ were recorded. In this paper, we discuss results for variants for which at least 100 genes were detected as differentially expressed at a false discovery rate (FDR) of 0.01 according to the method of Benjamini-Hochberg ${ }^{22}: 4$ cCNAs, 3 gene mutations and IGHV mutation status.

\section{Statistical analysis}

\section{Exploratory data analysis: PCA and clustering}

Statistical analyses were performed using $\mathrm{R}^{19}$ version 3.6. The exploratory data analysis was performed on data normalized and transformed using the variance stabilizing transformation (VST) provided by the DESeq2 package ${ }^{23}$. The 500 most variable genes were used in the principal component analysis (PCA) and hierarchical clustering. PCA was done using the prcomp function with scale. Hierarchical clustering with the ward.D2 method was performed on sample Euclidean distances computed on the scaled gene expression values. The complexHeatmaps package ${ }^{24}$ was used to visualize results.

\section{Batch effect estimation}

Transcriptome data were generated over a period of four years and platforms were changed with technological development during the period of sequencing, which led to changes in sequencing depth and read length (101, 125 and 151 nucleotides). Therefore, we considered the possibility of batch effects in the data due to platform differences ${ }^{25}$. Before adapter trimming we found a higher fraction of reads that contained adapter sequences in batches with longer reads. These resulted in batch dependent mapping to pseudogenes. After adapter trimming we did not detect differences in mapping towards pseudogenes or any 
associations between the top 10 principal components or the investigated genetic variants and different batches (Supplemental Figure S1).

\section{Differential expression analysis}

For each of the 23 genetic alterations (13 gene mutations, 10 CNAs) and the IGHV mutation status, differentially expressed genes were identified using the Gamma-Poisson generalized linear modeling (GLM) approach of DESeq2, version 1.16.1 $1^{23,26}$. Because of the large effects of IGHV mutation status and trisomy 12 on gene expression (as seen in the exploratory data analysis), these two variables were used as blocking factors in the models for each of the 22 remaining variants. In the model for IGHV mutation status, trisomy 12 was used as a blocking factor, and vice versa. Genetic interactions were identified by testing for an interaction term between the two variables IGHV mutation status and trisomy 12. Separately in each of these 25 DESeq2 analyses, the method of Benjamini and Hochberg ${ }^{22}$ was applied to account for multiple testing and control FDR of 0.01 .

\section{Gene set enrichment analysis}

Gene set enrichment analysis ${ }^{27}$ was performed using the R package clusterProfiler ${ }^{28}$ version 3.12 .0 based on ranked gene statistics from DESeq2. Hallmark and KEGG gene set collections version 4.0 were downloaded from $\mathrm{MSigDB}^{29}$. The significance of gene sets was determined using a permutation null $(\mathrm{B}=1000)$. $\mathrm{P}$-values were adjusted for multiple testing using the method of Benjamini and Hochberg ${ }^{22}$.

\section{Data Sharing Statement}

RNA-sequencing data are available at European Genome-phenomeArchive (EGA) under accession number EGAS00001001746. All code to reproduce this analysis is available at https://github.com/almutlue/transcriptome_cll (DOI:10.5281/zenodo.4600322). Analysis code and outputs are deployed as a browsable workflowr ${ }^{30}$ site: https://almutlue.github.io/transcriptome_cll/index.html. 


\section{Results}

\section{Unsupervised analysis reveals major drivers of gene expression variability}

We generated RNA-sequencing data from 184 CLL patient samples. To obtain a first overview of patterns of gene expression variability in CLL, we performed an unsupervised clustering analysis based on the 500 most variable genes (Figure 1A). This analysis showed a separation of distinct subgroups that largely coincided with IGHV mutation status/methylation epitype and the presence of trisomy 12. The role of IGHV mutation status and trisomy 12 was also reflected in the number of differentially expressed (DE) genes ( $>3000 \mathrm{DE}$ genes) (Figure 1B). A similar separation was seen in a principal component analysis (PCA) (Figure 1C,D). The first principal component, which represented $11 \%$ of the variance, was associated with IGHV mutation status, while the second component separated samples based on trisomy 12. These results indicate that these two genetic variables shape gene expression in CLL to a previously unappreciated extent. We also considered a classification of CLL based on global DNA methylation levels into three groups ${ }^{20,21}$, a refinement of the binary grouping by IGHV mutation status (Figure 1E). Accordingly, the first principal component arranged the DNA methylation subgroups into the order low, intermediate and high programmed (LP, IP, HP). These results indicate that even though the three groups classification was discovered using DNA methylation data, it is equally apparent at the level of gene expression. Indeed, the global gene expression patterns shown in Figure 1 imply a further refinement into five major groups, namely LP, IP and HP each with and without trisomy 12 .

The results of our unsupervised clustering analysis differ from those of a previous gene expression study, which also used unsupervised clustering of RNA-sequencing data to find novel subgroups of CLL termed $\mathrm{C} 1 / \mathrm{C} 2$, marked by 600 differentially expressed genes and associated with $\mathrm{BcR}$ activation and outcome ${ }^{10}$. In our data, hierarchical clustering of the samples based on the measurements of these 600 genes only, indeed resulted in two main clusters. However, most of these genes showed low variability across samples and only 26 of them were among the 500 most variable genes (Supplemental Figure S2). 


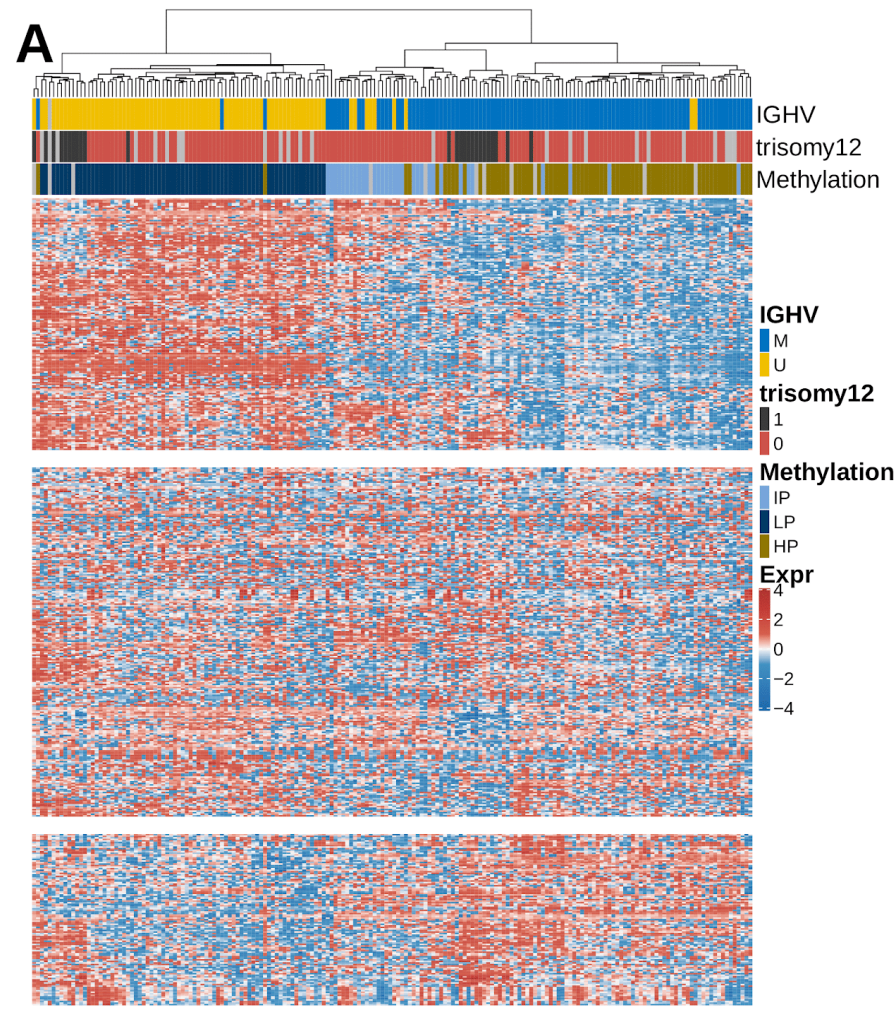

\section{B}
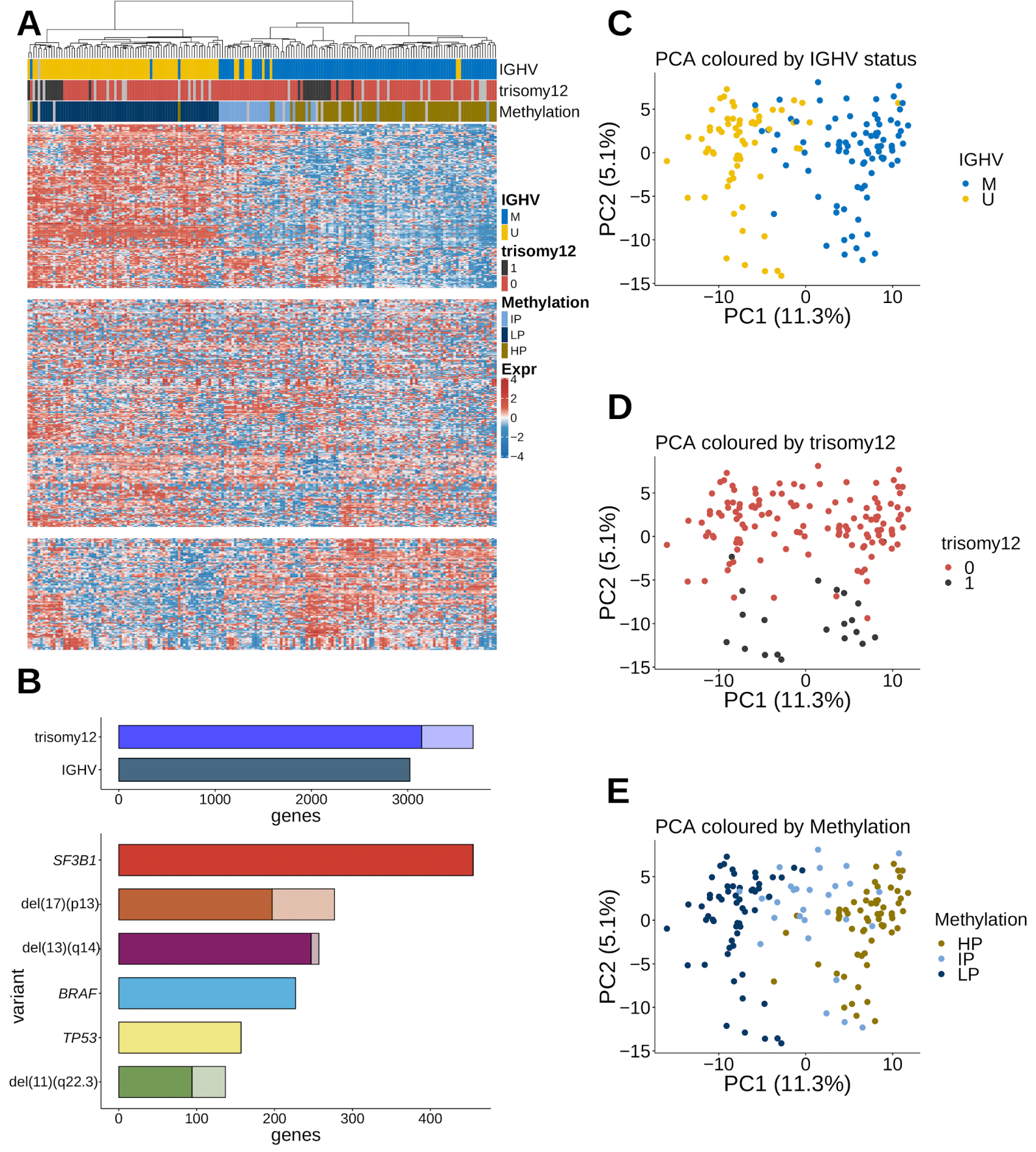

Figure 1. Gene expression variability in CLL: A) Heatmap of the gene expression counts. Samples (columns) are ordered in agreement with the hierarchical clustering based on the 500 most variable genes. Gene (rows) counts are row-centered log transformed (base 2). IGHV mutation status, methylation subgroups and trisomy 12 align with the clustering result. B) Number of differentially expressed genes (adjusted P-values $<0.01$ ) for genomic markers in CLL. Lighter colors indicate genes located on the same chromosome as the respective genetic lesion (potential dosage effects). C) IGHV status is associated with the first principal component, which explains $11.3 \%$ of the variance. D) Trisomy 12 is associated with the second principal component, which explains $5.1 \%$ of the variance. E) Methylation subgroups split up along principal component 1. 


\section{Mutations modulate gene expression in CLL}

We performed differential expression analysis to explore the effect of 23 recurrent genetic aberrations and the IGHV mutations status (Supplemental Figure S3). In total, we found 6 additional variants (besides trisomy 12 and the IGHV status) associated each with more than 100 differentially expressed genes. These were $\operatorname{del}(13)(\mathrm{q} 14), \operatorname{del}(17)(\mathrm{p} 13), \operatorname{del}(11)(\mathrm{q} 22)$, and SF3B1, TP53 and BRAF mutations (Figure 1B). Complete tables are provided in the computational analysis transcript.

We compared previous findings from the literature for single genetic aberrations with differentially expressed genes in this study. Mutations in the splicing factor SF3B1 gene showed more than 400 associations. Gene sets enriched in CLL with SF3B1 mutations included "Cytokine-cytokine receptor interaction" and "Phosphatidylinositol signaling system" (Supplemental Figure S4A). Among differentially expressed genes, we found the chaperone gene UQCC1 (Supplement Figure S4B), which has already been linked to SF3B1 mutations by differential isoform usage ${ }^{31}$. We also found PSD2, SRRM5 and TNXB (Supplemental Figure S3C-E) associated with SF3B1 mutation. TP53 is another commonly mutated gene in CLL and associated with inferior survival ${ }^{1}$. Differentially expressed genes in samples with TP53 mutation were enriched in “Oxidative phosphorylation” and "p53 signaling pathway" (Supplement Figure S5A). The transcriptional regulator $C D K 12$ is upregulated in TP53 mutated samples (Supplement Figure S4B). Further associations with TP53 include PGBD2, HYPK and the p53 antagonist MDM2 $2^{32}$ (Supplement Figure S4C-E).

\section{IGHV mutation status is linked to distinct gene expression changes}

The highest number of differentially expressed genes was found in the comparison between IGHV-mutated (M-CLL) and U-CLL: 3275 genes. This result is in agreement with the PCA of Figure 1C and shows that IGHV mutation status is the main determinant of gene expression variability in CLL. It implies a much larger impact of IGHV status on the transcriptome than previously detected $\left(11.3 \%\right.$ instead of $\left.1.5 \%{ }^{10}\right)$ and is in line with the key impact of IGHV mutation status on clinical course and biology of disease ${ }^{7-9}$. Genes previously found to be markers related to IGHV mutation status, including CD38, LPL, ZAP70, SEPT10, ADAM29 and PEG10 $0^{33-35}$, were also detected in our analysis (Supplemental Table 1).

To understand the pathways involved in U-CLL and M-CLL, we performed gene set enrichment analysis. Differentially expressed genes between IGHV groups were enriched in BcR signaling, T-cell receptor 
signaling and chemokine signaling pathways (Figure 2A). Within the BcR signaling gene set, we identified

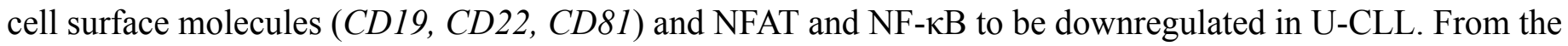
“T cell receptor signaling" gene set, ZAP70, PAK and p38 were upregulated in U-CLL, while IL10 and SHP1 were downregulated. Within chemokine signaling pathways, we found downregulation of $C X C R 3$ and CXCR4 in U-CLL, while a set of interferons (IFNB1, INFA21) were upregulated.

IGHV genes were also found among the most differentially expressed genes, but showed heterogeneous expression within the U-CLL and M-CLL groups. As expected, commonly used IGHV genes (IGHV1-69 or IGHV4-34) were associated with U-CLL and M-CLL, respectively. Gene expression showed a strong relation to IG gene usage and it's variant's expression (Figure 2B). These data show that RNA-sequencing can be used to assess IG gene usage. Further genes associated with IGHV groups were BCAT1, EGR3 and ZAP70 (Figure 2C-E)

In summary, our data are in line with the major biological role of IGHV mutation status in CLL and provide a resource to identify deregulated pathways in the disease.

\section{Intermediate programmed methylation group forms an independent gene expression}

\section{cluster}

Based on the global DNA methylation pattern, the stratification of CLL by IGHV mutation status has recently been refined by introducing a categorization into LP, IP, and HP programmed samples, which are thought to represent the cell of origin ${ }^{20,21}$. Based on gene expression data, we found these three groups along the first principle component. The IP group was placed between the LP and HP groups (Figure 1E). Thus, even though the groups were discovered on the basis of DNA methylation, a strong separation was found on the basis of unsupervised PCA of the gene expression data. Previous analysis of methylation groups in CLL suggested a disease-specific role of the transcription factors EGR, NFAT, API and EGF by establishing aberrant methylation patterns ${ }^{20}$. In line with this, we found NFATC1 and EGR1 among genes whose expression patterns were associated with methylation groups (Supplemental Figure S6A-B). A detailed analysis of the intermediate methylated subgroup revealed single genes including SOXI1 and MSI2 that were specific for this subgroup (Supplement Figure S6C-D). SOX11 is a transcription factor, which expression has been associated with adverse prognostic markers in CLL ${ }^{36}$. 


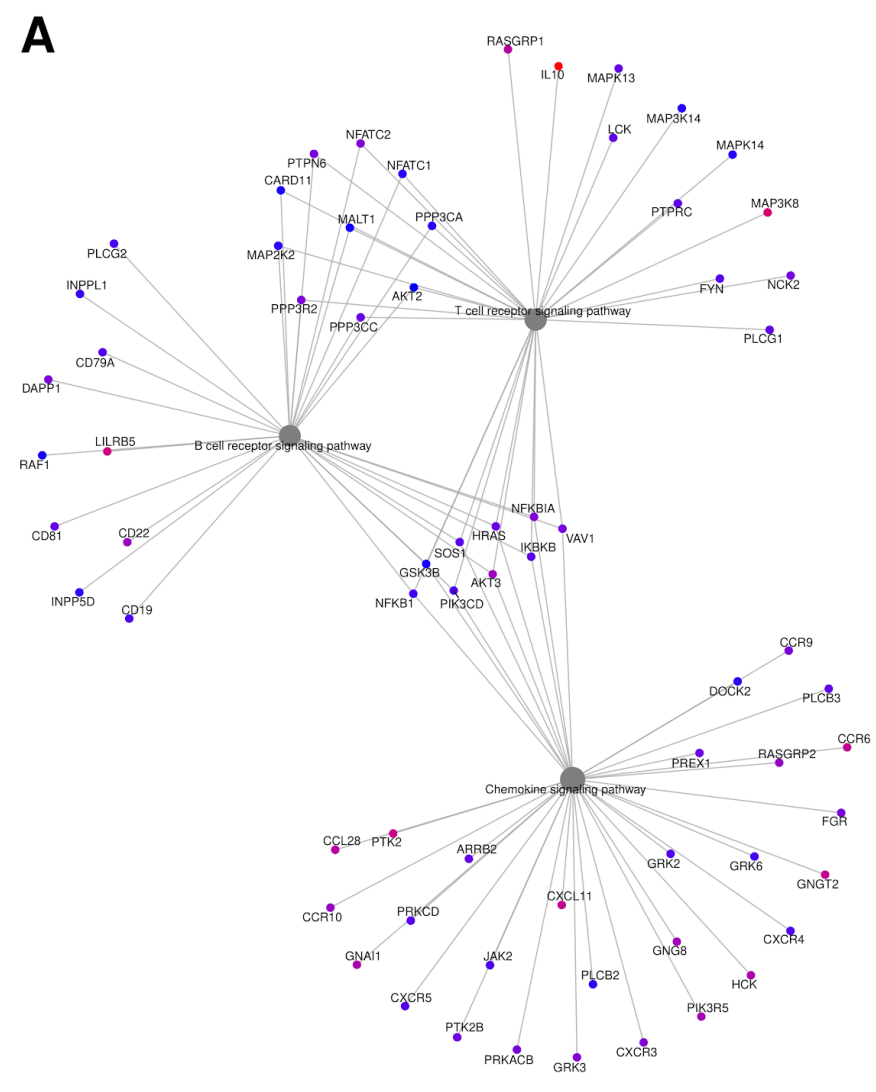

B
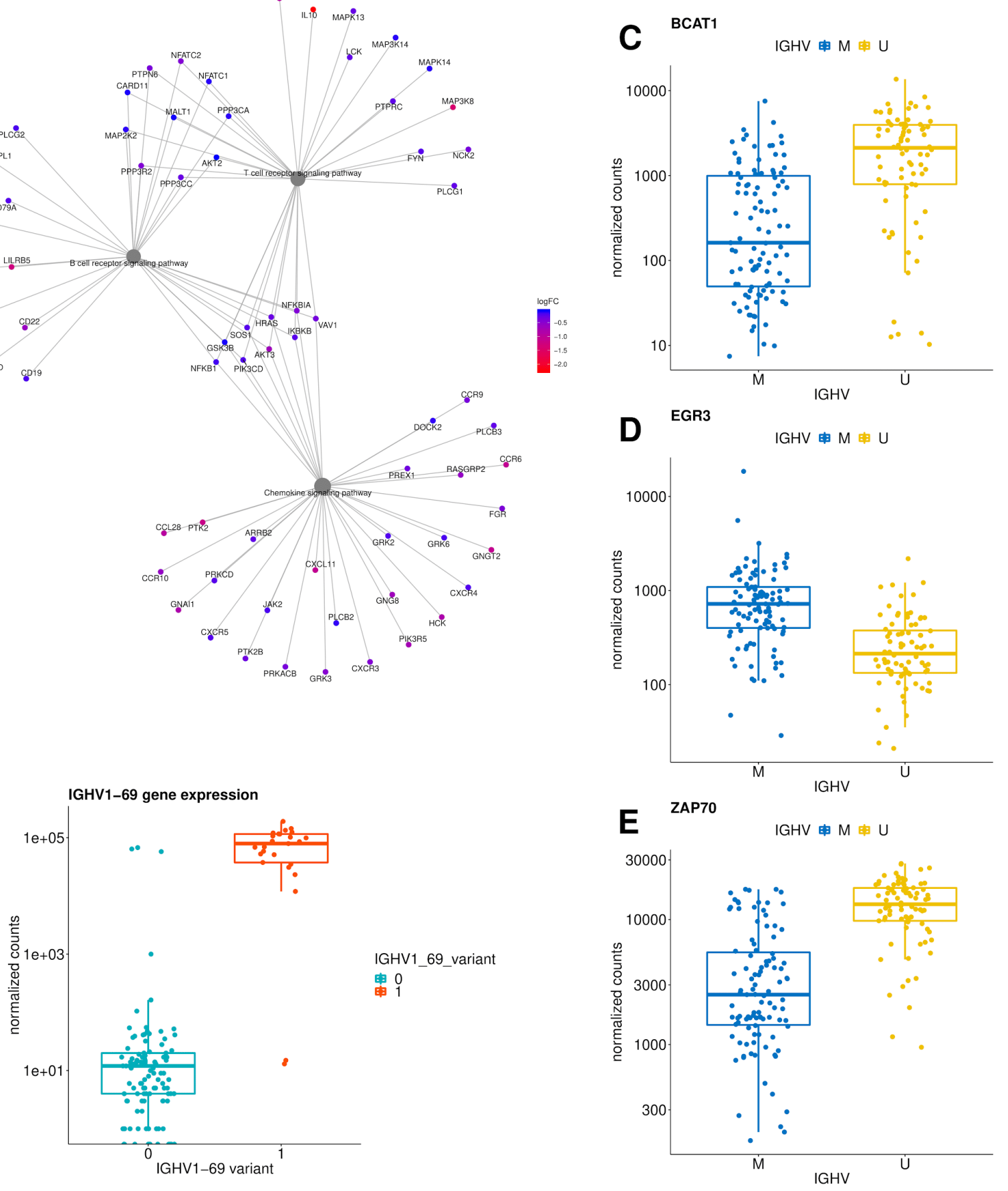

Figure 2, Gene expression changes between IGHV subgroups: A) Differentially expressed genes in enriched KEGG pathways for IGHV. B) IGHV1-69 expression by corresponding IGHV1-69 gene usage determined by IG gene analysis. C-E) Normalized gene counts for BCAT1, EGR3 and ZAP70 separated by IGHV mutation status. 


\section{Expression signature in CLL with trisomy 12}

We identified over 3000 differentially expressed genes (with adjusted P-value $<0.01$ ) in CLL with trisomy 12 (Figure 1B). Even though chromosome 12 harbours many upregulated genes, the majority of all differentially expressed genes were on other chromosomes and therefore cannot be ascribed to a simple gene dosage effect (Figure 3A).

Among the differentially expressed genes, we found numerous genes involved in chemokine signaling such as VAV1 (Figure 3B,C). Chemokine signaling pathways are induced by chemokine binding and activate MAPK signaling ${ }^{37,38}$. In line with this, we identified differentially expressed genes enriched in MAPK signaling. We also detected an enrichment for the mTOR-signaling pathway, a known modulator of chemokine signaling $^{39}$ (Figure 3B). Consistent with previous reports, integrins like ITGAL, ITGB2 and ITGA4 were also upregulated in trisomy 12 samples $^{13}$ (Supplemental table 1). We also found the immune checkpoint gene CTLA4 (Figure 3D) downregulated in trisomy 12 samples. CTLA4 has previously been linked to CLL and is associated with increased proliferation and tumor progression ${ }^{40,41}$.

A known mechanism of tumor cells to escape the immune system is to inhibit tumor-specific T cells, and support the conversion of anti-tumor type 1 macrophages to pro-tumor type 2 macrophages by upregulation of ecto-5'-nucleotidase (NT5E), which is necessary to convert extracellular ATP into adenosine. A previous study on gene expression in trisomy 12 patients identified $N T 5 E$ as an important element in a trisomy 12 expression network model, but did not directly measure its expression ${ }^{13}$. In our study, we found higher expression of NTSE in trisomy 12 and thus can confirm this hypothesis of Abruzzo et al. ${ }^{13}$ (Figure 3E).

Altogether, these results suggest that modulation of MAPK-signaling signaling through chemokine signaling and mTOR-signaling are important mechanisms in trisomy 12 tumorigenesis. 
A

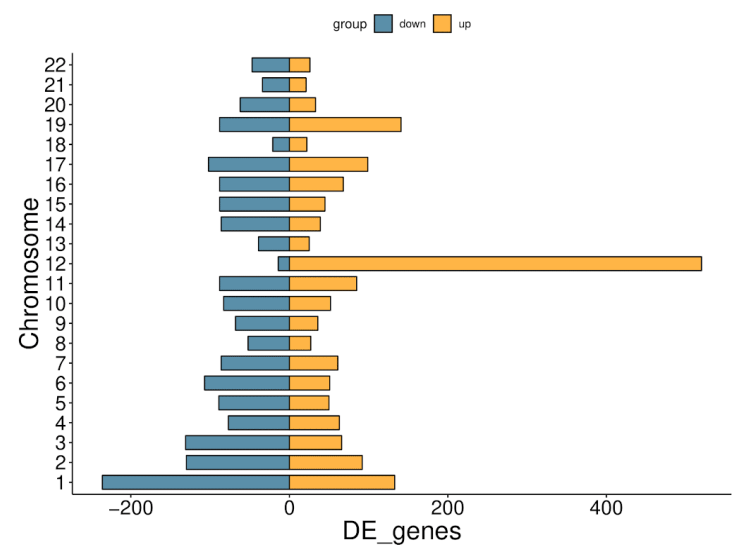

B

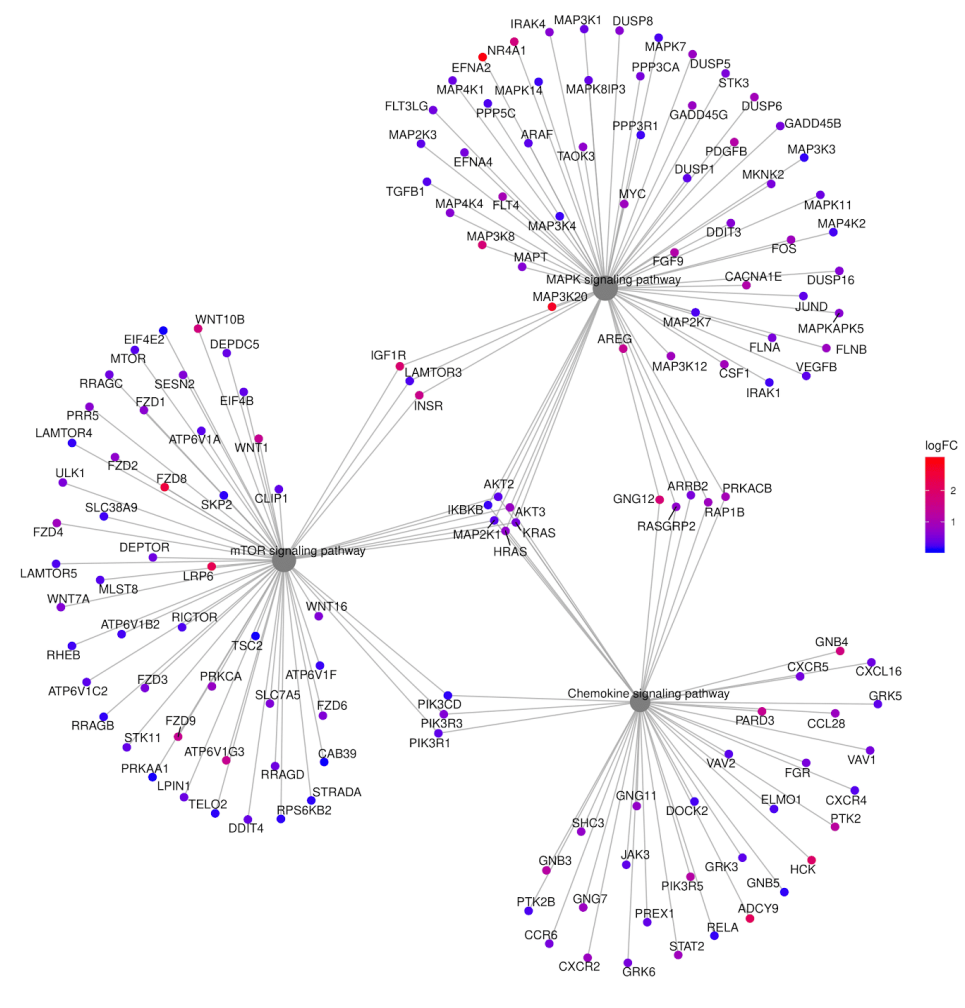

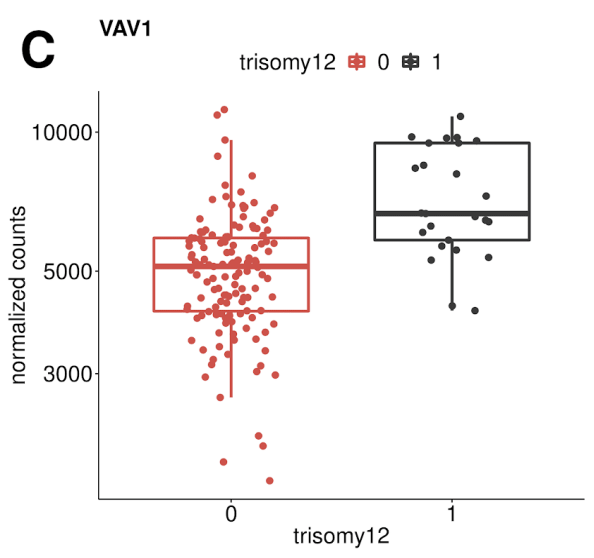
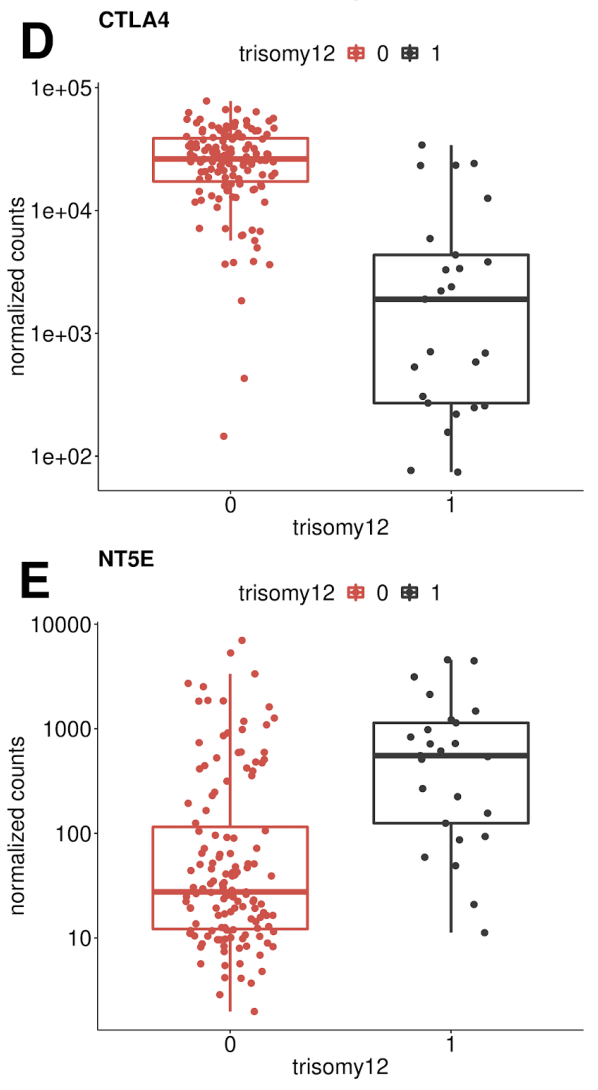

Figure 3, Gene expression in CLL with trisomy 12: A) Role of dosage effect: chromosomal distribution of DE genes in CLL with trisomy 12. Chromosome 12 has the highest number of DE genes, but the majority of DE genes is distributed across all chromosomes and cannot be ascribed to a dosage effect. B) DE genes in enriched KEGG pathways of trisomy 12. C-E) Normalized gene counts of VAV1, CTLA4 and NTSE. 


\section{IGHV status and trisomy 12 affect gene expression in an epistatic way}

Epistasis describes a phenomenon where the effect of a genetic variant is dependent on the presence or absence of another genetic variant ${ }^{42}$. Although it is thought to be prevalent, there is almost no data on epistasis between cancer mutations. Because of the major effects of each of these variants individually, we asked whether and to what extent epistatic interactions existed between IGHV mutation status and trisomy 12. We fit a generalized linear model (DESeq2) with main and interaction effects for these covariates. Significant interactions were detected for 893 genes (10\% FDR). For these genes, the effect of trisomy 12 was different in U-CLL and M-CLL. We observed four distinct types of epistatic interactions ${ }^{43,44}$ and classified the 893 genes into these categories (Figure 4A-D): synergy, where the samples with both variants showed a stronger up-regulation than expected from the single variants; buffering, when the presence of both variants led to a strong reduction of gene expression; inversion, when the effects in the single variants were reversed in the double variant; suppression, when a strong expression change (up or downregulation) of a gene in a single variant was absent in samples with both variants (Figure 4E, G,). Figure 4A-D shows the count data for exemplary genes. Early B-cell factor $1(E B F-1)$ is up-regulated in all trisomy 12 cases, but this effect is on average about 100 times stronger in M-CLL patients compared to U-CLL patients (synergy) (Figure 4A). While fibroblast growth factor $2(F G F 2)$ is consistently upregulated in trisomy 12 cases with U-CLL, this effect is reversed in M-CLL (suppression) (Figure 4B). Lymphoid enhancer binding factor 1 (LEF-1) shows a stable gene expression across samples and has been suggested and tested as a clinical marker for CLL ${ }^{45}$. While the presence of either one of the genetic variants (IGHV-M or trisomy 12) does not seem to have an effect, samples with both of them express consistently lower levels of LEF1 (buffering) (Figure 4D). These effects cannot be explained by looking at the genotypes independently or by modeling an additive effect of the variants. Each of these interaction types affected dozens or hundreds of genes, and we asked whether there were underlying biological functions. We used gene set enrichment analysis on each of the four types of epistasis, as well as on the combined set of all 893 genes. The overall set of genes with an epistasis expression pattern was enriched in TNF alpha signaling via NF-kB, MYC targets and IL2/STAT5 signaling. A recent study linked NF-kB and EBF1 expression with reduced levels of B-cell signaling ${ }^{46}$. Both IGHV status and trisomy 12 are known to affect BcR signaling. In the type-specific analysis, these pathways were still significantly enriched. In addition, we found the G2M checkpoint pathway enriched in the set of buffered genes, as well as in the set of inversion genes (Figure 4F). 
bioRxiv preprint doi: https://doi.org/10.1101/2021.04.16.440134; this version posted April 16, 2021. The copyright holder for this preprint (which was not certified by peer review) is the author/funder, who has granted bioRxiv a license to display the preprint in perpetuity. It is made available under aCC-BY-NC-ND 4.0 International license.

A IGHV_tri12 申 IGHV-UM 申 IGHV-M 申 trisomy12 申 both $\mathbf{E}$

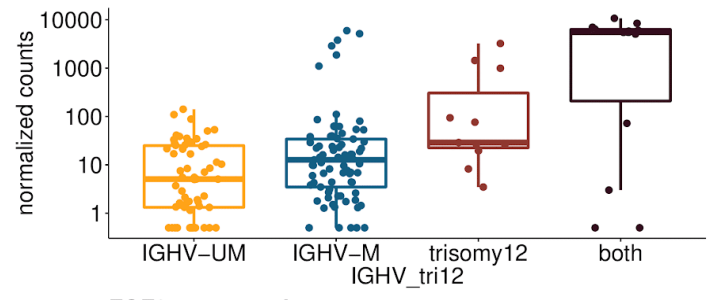

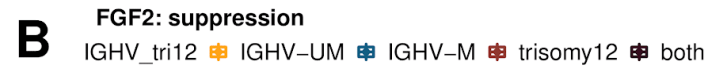
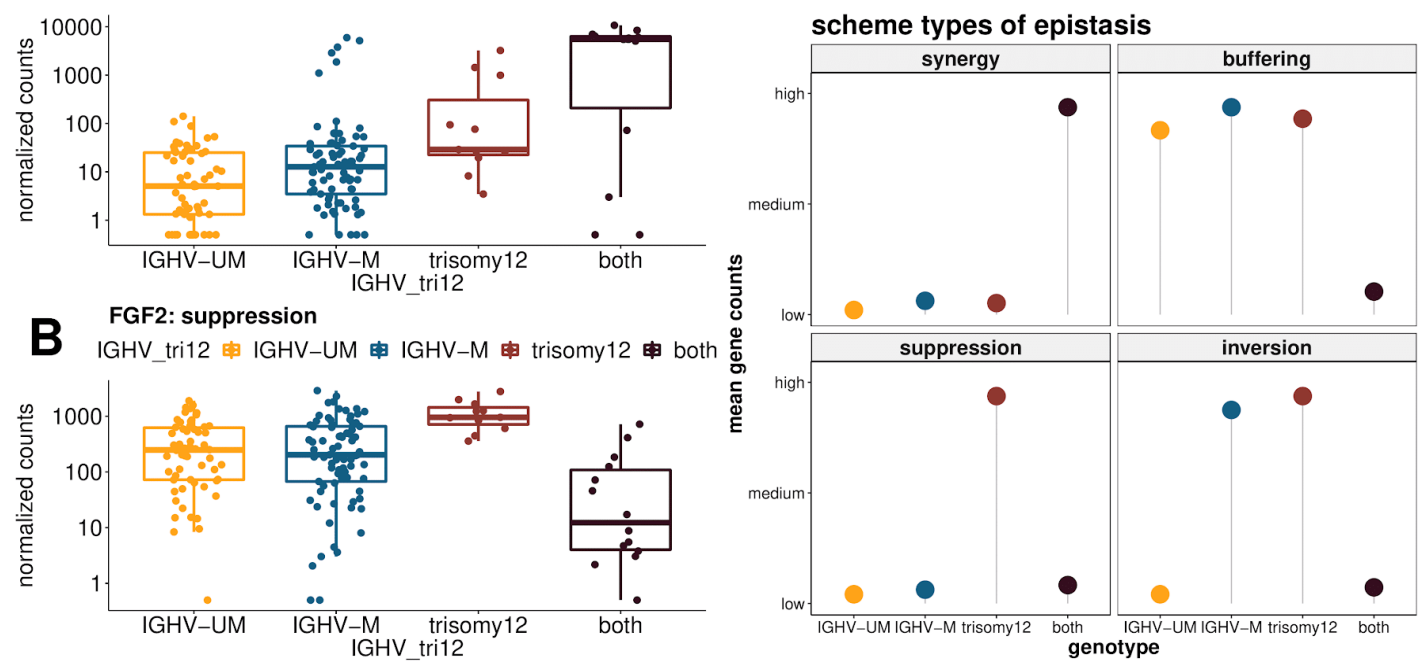

C SYBU: inversion

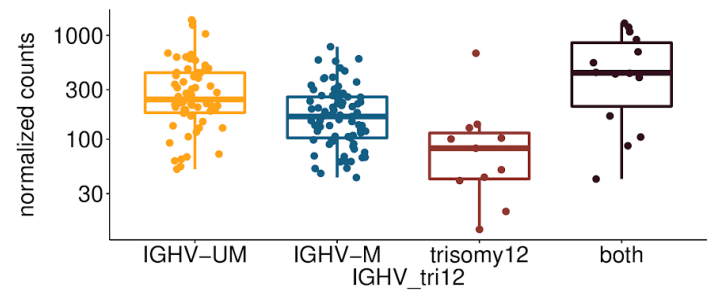

G

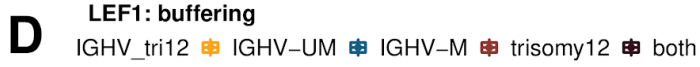

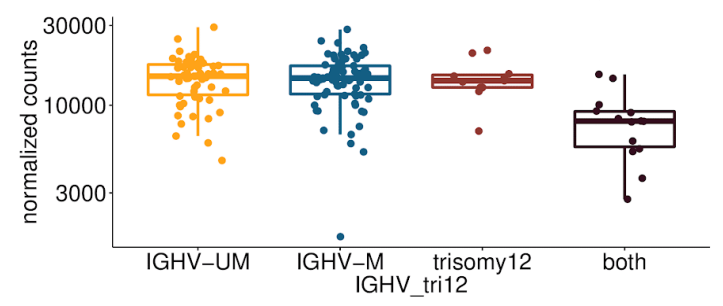

$\mathbf{F}$
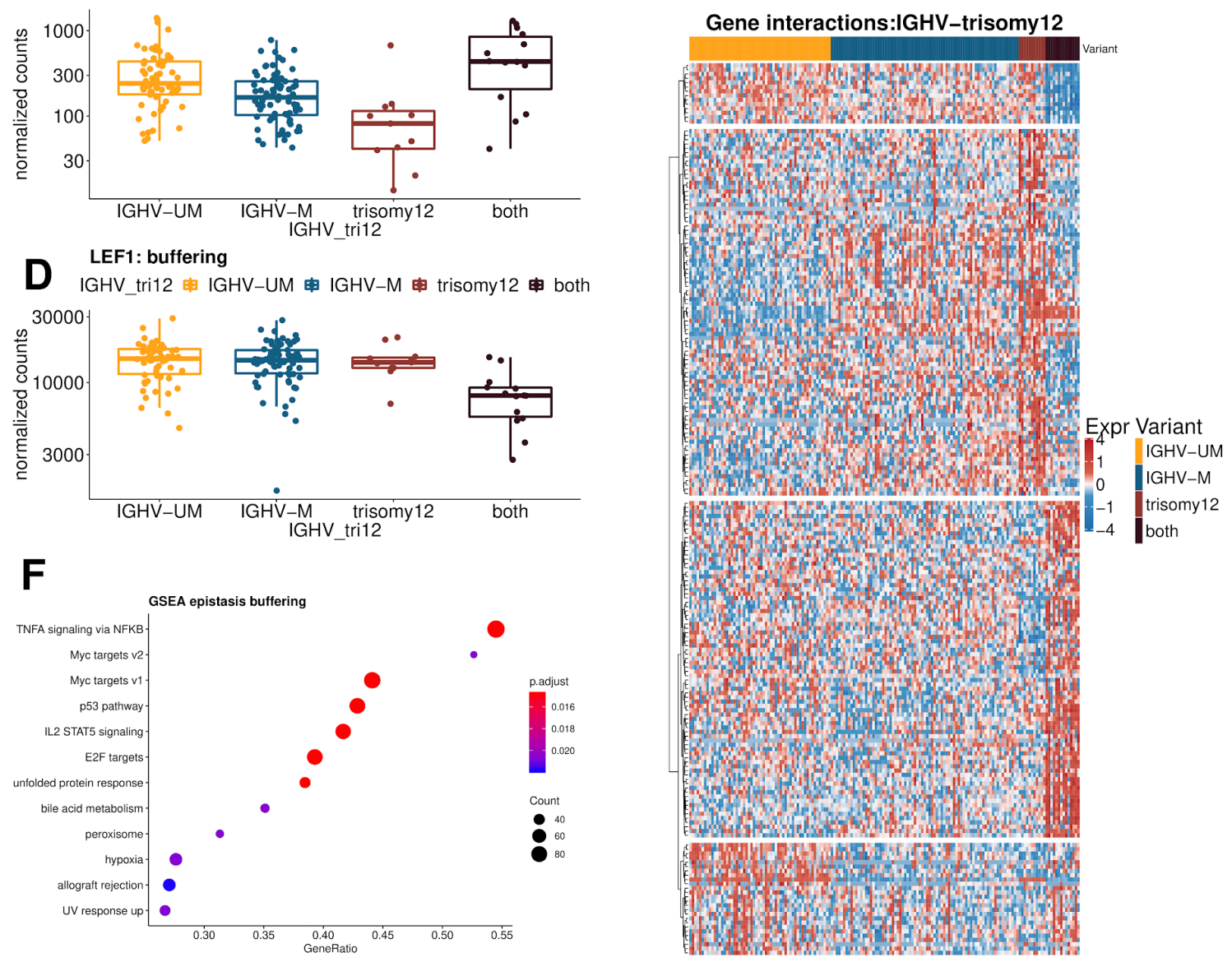

Figure 4. Mixed epistasis of trisomy 12 and IGHV mutation status: A-D) Types of gene expression epistasis: $E B F 1$ (synergy), FGF2 (suppression), SYBU (inversion), LEF1 (buffering) E) Schematic classification of epistasis F) Enriched pathways in genes with a buffering epistasis. G) Expression of epistatic gene interactions between trisomy 12 and M-CLL (adjusted P-value $<0.1$ ). 


\section{The epistatic interaction between IGHV status and trisomy 12 affects ex vivo drug response in CLL}

Ex vivo sensitivity to drugs is an informative cellular phenotype that reflects pathway dependencies of CLL cells. We asked whether the epistatic interaction between IGHV mutation status and trisomy 12 on expression level also affected the drug response phenotype. Previously, we measured the ex vivo sensitivity, as measured by cell viability, of our $184 \mathrm{CLL}$ samples towards 63 compounds $^{47}$. Again using two-way ANOVA with an interaction term, we identified 6 drugs for which there was a significant (10\% FDR) interaction between IGHV mutation status and trisomy 12 (Figure 5). For four drugs, namely, vorinostat, NU7441, fludarabine and AZD7762, we observed a suppression effect, where trisomy 12 led to increased drug sensitivity in the U-CLL, but not the M-CLL samples. For the other two drugs, chaetoglobosin A and BIX02188, the samples with trisomy 12 showed increased resistance particularly in M-CLL, but not in U-CLL.

The four drugs with the suppression phenotype directly or indirectly target DNA: NU7441 inhibits DNA-dependent protein kinase (DNA-PK) and therefore potentiate DNA double-strand breaks ${ }^{48}$; AZD7762 is a checkpoint kinase (CHEK) inhibitor, which can impair DNA repair and increases cell death ${ }^{49}$; fludarabine directly inhibits DNA synthesis and disrupt cell cycle ${ }^{50}$; vorinostat, a histone deacetylase (HDAC) inhibitor, was also reported to induce reactive oxygen species and DNA damage in leukemia cells ${ }^{51}$. 

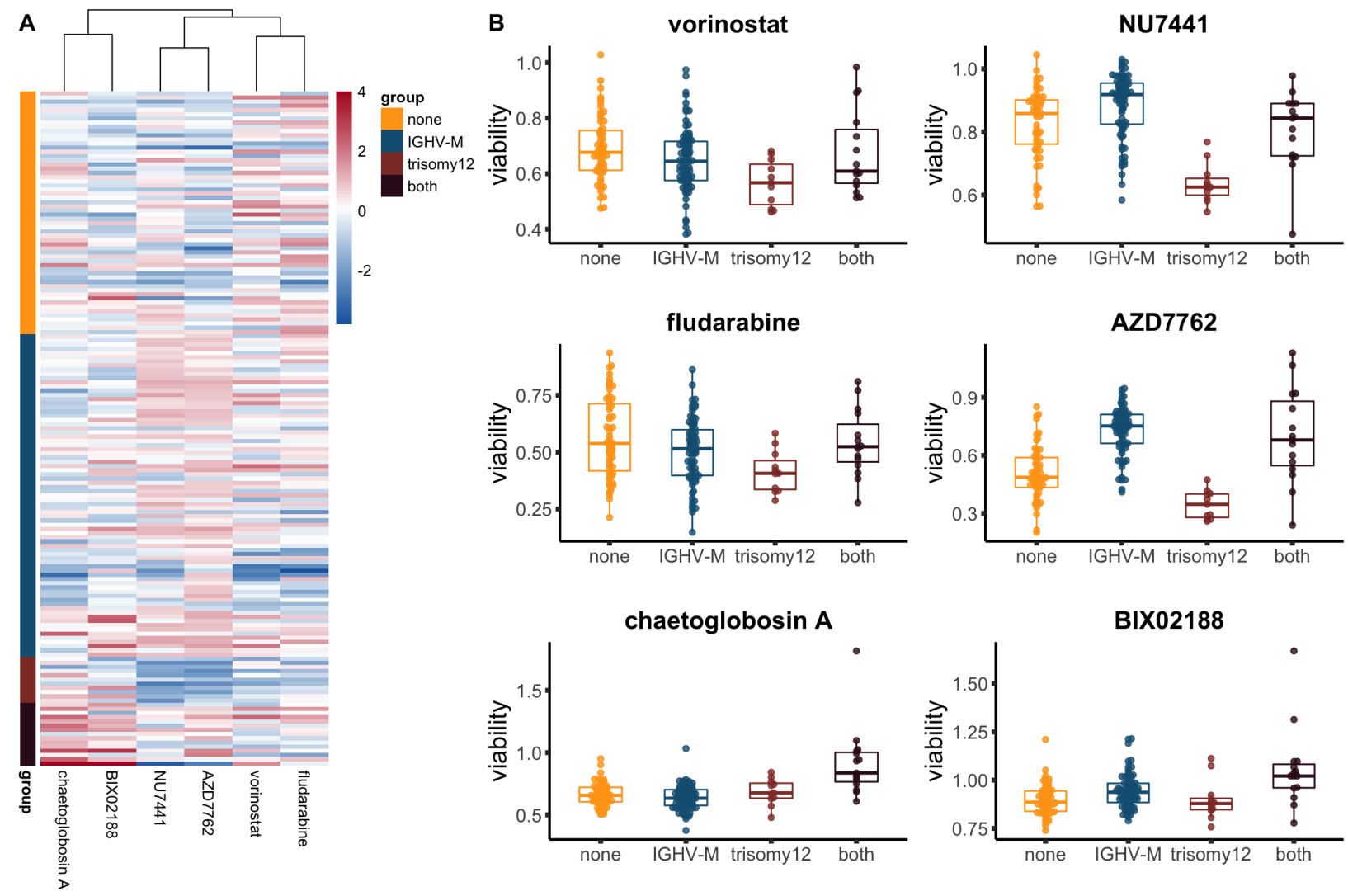

Figure 5. Ex vivo drug response phenotype related to the epistatic interaction between IGHV status and trisomy 12, A) Heatmap plot showing the responses of CLL samples (rows) towards the six drugs (columns) for which there was a significant interaction between IGHV mutation status and trisomy 12. The coloring encodes the column-wise z-scores of sample viabilities after drug treatment. B) Boxplots of the viabilities (normalized to DMSO controls) of CLL samples, stratified by their IGHV and trisomy 12 status, towards the six drugs shown in the heatmap.

\section{Discussion}

We analyzed 184 CLL transcriptomes, identified gene expression signatures for the most prevalent genetic aberrations and show that these can be used to point towards underlying pathways and potential pathobiological mechanisms. The IGHV mutation status, the three DNA methylation subgroups and trisomy 12 were found as the main drivers of gene expression variability in CLL. This is evident both in unsupervised analyses (clustering, PCA) and in supervised differential expression analysis. Notably, we revealed a much higher impact of IGHV mutation status on the CLL transcriptome than previous reports ${ }^{10}$. The disease stratification by IGHV status is further refined by the three DNA methylation subgroups. We identified genes whose expression follows an apparent continuum from LP, IP to HP in CLL. This finding supports the 
biological relevance of these three groups and suggests that although they were discovered based on DNA methylation, they are similarly evident at the level of gene expression. Altogether, these results highlight the potential of gene expression profiling to increase our understanding of CLL.

To avoid potential confounding effects of multiple aberrations, other studies have focused on samples with single abnormalities. While this approach could successfully resolve trisomy 12 specific gene expression, it is limited to only a subset of the disease and to selected variants ${ }^{13}$. Here, we demonstrate an improved approach that employs differential expression analysis with generalized linear models and blocking factors, and that is able to use the full range of CLL and to investigate a larger number of genetic aberrations.

Genetic interactions, or epistasis, where the effect of one mutation depends on the genetic status of another locus, is a well known concept in genetics. However, there is surprisingly little data on such phenomena in cancer. Hence, we used the opportunity to study the combinatorial effects of trisomy 12 and the IGHV mutation status on gene expression variability. We identified a large number of genes ( 900) whose expression depended on the presence or absence of either of these two aberrations in a non-additive manner. We categorized these genes into four categories: buffering, synergy, suppression and inversion, each of which contained dozens to hundreds of genes. This means that there is not a single, simple epistasis phenotype between these two aberrations, but a complex, "mixed" pattern. Mixed epistasis of the gene expression phenotypes of pairs of gene alterations has been described in a yeast model system ${ }^{44}$. We employed the genetic interactions between IGHV mutation status and trisomy 12 on gene expression phenotypes to identify pathways, including TNF alpha signaling via NF- $\kappa B$ and the G2M checkpoint pathway, that mediate the effects of these variants. Our results raise the question whether the pathobiology of trisomy 12 may be different between U-CLL and M-CLL, which could be explained by more active BcR signaling in U-CLL and a more diverse set of signaling initiators (independent of the BcR) in M-CLL, and can help us to understand subtype-specific differences.

To our knowledge, this study is the first to provide evidence for this concept in humans, and in cancer. Both the phenomenon itself of epistasis between cancer driving and/or constitutive mutations, and the fact that it can be 'mixed' (follow different patterns) for different phenotypes form a new layer of complexity in CLL and in tumor biology more generally. Hence, our study highlights the inherent limitations of studying individual cancer genetic lesions, and points to the need to also map out and understand how they interact and modify each other's effects. 


\section{Additional Files}

Supplementary Table S1: patient_overview.xlsx

Supplementary Table S2: de_genes_all.xlsx

\section{Acknowledgments}

We thank members of the Huber and Zenz research teams for valuable discussions. The work was supported by the European Union (Horizon 2020 project SOUND under grant agreement number 633974) and the German Federal Ministry of Education and Research (TRANSCAN project GCH-CLL 143 under grant agreement number 01KT1610 and CompLS project MOFA under grant agreement number 031L0171A). T. Zenz was supported by the UZH Clinical Research Priority Program "Next Generation Drug Response Profiling for Personalized Cancer Care ", the Swiss Cancer League (KFS-4439-02-2018), and the Monique-Dornonville-de-la-Cour Stiftung. R. Rosenquist received funding from the Swedish Cancer Society, the Swedish Research Council, the Knut and Alice Wallenberg Foundation, and Radiumhemmets Forskningsfonder, Stockholm. For technical support and expertise, we thank the DKFZ Genomics and Proteomics Core Facility. We thank Hanno Glimm, Stefan Fröhling, Daniela Richter, Roland Eils, Peter Lichter, Stephan Wolf, Katja Beck, and Janna Kirchhof for infrastructure and program development within DKFZ- HIPO and NCT POP.

\section{Authorship Contributions}

A Lütge: conceptualization, data curation, formal analysis, visualization, methodology, and writing - original draft, review, and editing.

J. Lu: conceptualization, formal analysis, methodology, and writing - original draft, review, and editing.

J. Hüllein: data curation

T. Walther: data curation

L. Sellner: data curation, - review, and editing

B. Wu: data curation, - review, and editing

R. Rosenquist: data curation, writing - review, and editing 
C. Oakes: data curation, - review, and editing

S. Dietrich: data curation, - review, and editing

W. Huber: conceptualization, supervision, methodology, project administration, and writing - original draft, review, and editing.

T. Zenz: conceptualization, supervision, methodology, project administration, and writing - original draft, review, and editing.

\section{Conflict of Interest Disclosures}

RR has received honoraria from Abbvie, AstraZeneca, Janssen, Illumina and Roche; LS is currently an employee of Takeda; the remaining authors declare no competing interests.

\section{References}

1. Campo E, Cymbalista F, Ghia P, et al. TP53 aberrations in chronic lymphocytic leukemia: an overview of the clinical implications of improved diagnostics. Haematologica. 2018;103(12):1956-1968.

2. Rossi D, Gaidano G. ATM and chronic lymphocytic leukemia: mutations, and not only deletions, matter. Haematologica. 2012;97(1):5-8.

3. Rossi D, Rasi S, Fabbri G, et al. Mutations of NOTCH1 are an independent predictor of survival in chronic lymphocytic leukemia. Blood. 2012;119(2):521-529.

4. Stevenson FK, Krysov S, Davies AJ, Steele AJ, Packham G. B-cell receptor signaling in chronic lymphocytic leukemia. Blood. 2011;118(16):4313-4320.

5. Zenz T, Mertens D, Küppers R, Döhner H, Stilgenbauer S. From pathogenesis to treatment of chronic lymphocytic leukaemia. Nat. Rev. Cancer. 2010;10(1):37-50.

6. Fabbri G, Dalla-Favera R. The molecular pathogenesis of chronic lymphocytic leukaemia. Nat. Rev. Cancer. 2016;16(3):145-162.

7. Rosenquist R, Ghia P, Hadzidimitriou A, et al. Immunoglobulin gene sequence analysis in chronic lymphocytic leukemia: updated ERIC recommendations. Leukemia. 2017;31(7):1477-1481.

8. Damle RN, Wasil T, Fais F, et al. Ig V gene mutation status and CD38 expression as novel prognostic indicators in chronic lymphocytic leukemia. Blood. 1999;94(6):1840-1847.

9. Hamblin TJ, Davis Z, Gardiner A, Oscier DG, Stevenson FK. Unmutated Ig V(H) genes are associated with a more aggressive form of chronic lymphocytic leukemia. Blood. 1999;94(6):1848-1854. 
10. Ferreira PG, Jares P, Rico D, et al. Transcriptome characterization by RNA sequencing identifies a major molecular and clinical subdivision in chronic lymphocytic leukemia. Genome Res. 2014;24(2):212-226.

11. Rosenwald A, Alizadeh AA, Widhopf G, et al. Relation of gene expression phenotype to immunoglobulin mutation genotype in B cell chronic lymphocytic leukemia. J. Exp. Med. 2001;194(11):1639-1647.

12. Dvinge H, Ries RE, Ilagan JO, et al. Sample processing obscures cancer-specific alterations in leukemic transcriptomes. Proc Natl Acad Sci USA. 2014;111(47):16802-16807.

13. Abruzzo LV, Herling CD, Calin GA, et al. Trisomy 12 chronic lymphocytic leukemia expresses a unique set of activated and targetable pathways. Haematologica. 2018;103(12):2069-2078.

14. Herling CD, Coombes KR, Benner A, et al. Time-to-progression after front-line fludarabine, cyclophosphamide, and rituximab chemoimmunotherapy for chronic lymphocytic leukaemia: a retrospective, multicohort study. Lancet Oncol. 2019;20(11):1576-1586.

15. Unable to find information for 6085286.

16. Wingett SW, Andrews S. FastQ Screen: A tool for multi-genome mapping and quality control. [version 2; peer review: 4 approved]. F1000Res. 2018;7:1338.

17. Dobin A, Gingeras TR. Mapping RNA-seq Reads with STAR. Curr. Protoc. Bioinformatics. 2015;51:11.14.1-11.14.19.

18. Anders S, Pyl PT, Huber W. HTSeq — a Python framework to work with high-throughput sequencing data. Bioinformatics. 2015;31(2):166-169.

19. R: The R Project for Statistical Computing.

20. Oakes CC, Seifert M, Assenov Y, et al. DNA methylation dynamics during B cell maturation underlie a continuum of disease phenotypes in chronic lymphocytic leukemia. Nat. Genet. 2016;48(3):253-264.

21. Kulis M, Heath S, Bibikova M, et al. Epigenomic analysis detects widespread gene-body DNA hypomethylation in chronic lymphocytic leukemia. Nat. Genet. 2012;44(11):1236-1242.

22. Benjamini Y, Hochberg Y. Controlling the false discovery rate: A practical and powerful approach to multiple testing. Journal of the Royal Statistical Society: Series B (Methodological). 1995;57(1):289-300.

23. Love MI, Huber W, Anders S. Moderated estimation of fold change and dispersion for RNA-seq data with DESeq2. Genome Biol. 2014;15(12):550.

24. Gu Z, Eils R, Schlesner M. Complex heatmaps reveal patterns and correlations in multidimensional 
genomic data. Bioinformatics. 2016;32(18):2847-2849.

25. Leek JT, Scharpf RB, Bravo HC, et al. Tackling the widespread and critical impact of batch effects in high-throughput data. Nat. Rev. Genet. 2010;11(10):733-739.

26. Anders S, Huber W. Differential expression analysis for sequence count data. Genome Biol. 2010;11(10):R106.

27. Subramanian A, Tamayo P, Mootha VK, et al. Gene set enrichment analysis: a knowledge-based approach for interpreting genome-wide expression profiles. Proc Natl Acad Sci USA. 2005;102(43):15545-15550.

28. Yu G, Wang L-G, Han Y, He Q-Y. clusterProfiler: an R package for comparing biological themes among gene clusters. OMICS. 2012;16(5):284-287.

29. Liberzon A, Birger C, Thorvaldsdóttir H, et al. The Molecular Signatures Database (MSigDB) hallmark gene set collection. Cell Syst. 2015;1(6):417-425.

30. Blischak JD, Carbonetto P, Stephens M. Creating and sharing reproducible research code the workflowr way. [version 1; peer review: 3 approved]. F1000Res. 2019;8:1749.

31. Reyes A, Blume C, Pelechano V, et al. Mutated SF3B1 is associated with transcript isoform changes of the genes UQCC and RPL31 both in CLLs and uveal melanomas. BioRxiv. 2013;

32. Shangary S, Wang S. Targeting the MDM2-p53 interaction for cancer therapy. Clin. Cancer Res. 2008;14(17):5318-5324.

33. Kienle D, Benner A, Läufle C, et al. Gene expression factors as predictors of genetic risk and survival in chronic lymphocytic leukemia. Haematologica. 2010;95(1):102-109.

34. Rassenti LZ, Jain S, Keating MJ, et al. Relative value of ZAP-70, CD38, and immunoglobulin mutation status in predicting aggressive disease in chronic lymphocytic leukemia. Blood. 2008;112(5):1923-1930.

35. Benedetti D, Bomben R, Dal-Bo M, et al. Are surrogates of IGHV gene mutational status useful in B-cell chronic lymphocytic leukemia? The example of Septin-10. Leukemia. 2008;22(1):224-226.

36. Roisman A, Stanganelli C, Nagore VP, et al. SOX11 expression in chronic lymphocytic leukemia correlates with adverse prognostic markers. Tumour Biol. 2015;36(6):4433-4440.

37. Soriano SF, Serrano A, Hernanz-Falcón P, et al. Chemokines integrate JAK/STAT and G-protein pathways during chemotaxis and calcium flux responses. Eur. J. Immunol. 2003;33(5):1328-1333.

38. Cuesta-Mateos C, López-Giral S, Alfonso-Pérez M, et al. Analysis of migratory and prosurvival pathways induced by the homeostatic chemokines CCL19 and CCL21 in B-cell chronic lymphocytic 
leukemia. Exp. Hematol. 2010;38(9):756-64, 764.e1.

39. Munk R, Ghosh P, Ghosh MC, et al. Involvement of mTOR in CXCL12 mediated T cell signaling and migration. PLoS ONE. 2011;6(9):e24667.

40. Mittal AK, Chaturvedi NK, Rohlfsen RA, et al. Role of CTLA4 in the proliferation and survival of chronic lymphocytic leukemia. PLoS ONE. 2013;8(8):e70352.

41. Oh YM, Kwon YE, Kim JM, et al. Chfr is linked to tumour metastasis through the downregulation of HDAC1. Nat. Cell Biol. 2009;11(3):295-302.

42. Fisher RA. The Correlation between Relatives on the Supposition of Mendelian Inheritance. Trans. R. Soc. Edinb. 1919;52(02):399-433.

43. van Wageningen S, Kemmeren P, Lijnzaad P, et al. Functional overlap and regulatory links shape genetic interactions between signaling pathways. Cell. 2010;143(6):991-1004.

44. Sameith K, Amini S, Groot Koerkamp MJA, et al. A high-resolution gene expression atlas of epistasis between gene-specific transcription factors exposes potential mechanisms for genetic interactions. $B M C$ Biol. 2015;13:112.

45. Menter T, Trivedi P, Ahmad R, et al. Diagnostic Utility of Lymphoid Enhancer Binding Factor 1 Immunohistochemistry in Small B-Cell Lymphomas. Am. J. Clin. Pathol. 2017;147(3):292-300.

46. Meijers RWJ, Muggen AF, Leon LG, et al. Responsiveness of chronic lymphocytic leukemia cells to B-cell receptor stimulation is associated with low expression of regulatory molecules of the nuclear factor- $\kappa$ B pathway. Haematologica. 2020;105(1):182-192.

47. Dietrich S, Oleś M, Sellner L, et al. Drug perturbation based stratification of lymphoproliferative disorders. Hematol. Oncol. 2017;35:56-56.

48. Unable to find information for 8530527.

49. Zabludoff SD, Deng C, Grondine MR, et al. AZD7762, a novel checkpoint kinase inhibitor, drives checkpoint abrogation and potentiates DNA-targeted therapies. Mol. Cancer Ther. 2008;7(9):2955-2966.

50. Ricci F, Tedeschi A, Morra E, Montillo M. Fludarabine in the treatment of chronic lymphocytic leukemia: a review. Ther. Clin. Risk Manag. 2009;5(1):187-207.

51. Petruccelli LA, Dupéré-Richer D, Pettersson F, et al. Vorinostat induces reactive oxygen species and DNA damage in acute myeloid leukemia cells. PLoS ONE. 2011;6(6):e20987. 